\title{
Incidência de caquexia, anemia e sintomas de impacto nutricional em pacientes oncológicos
}

\author{
Cachexia incidence, anemia and nutritional impact symptoms \\ in cancer patients
}

\author{
Leoni Aparecida da Rocha* \\ Mariana Abe Vicente Cavagnari* \\ Angélica Rocha de Freitas Melhem* \\ Gabriela Datsch Bennemann* \\ Lindsay Bianca Buzato Antunes** \\ Diogo Gavarrete** \\ Dalton Luiz Schiessel*
}

\begin{abstract}
Resumo
Sintomas de impacto nutricional (SIN) e anemia são alterações fisiológicas frequentemente observadas em pacientes oncológicos antes do diagnóstico e durante o tratamento da radioterapia e quimioterapia podendo interferir na condição geral do paciente e na incidência da caquexia do câncer. O objetivo deste estudo foi avaliar a incidência de caquexia, SIN e anemia em pacientes oncológicos. Foi avaliado o peso, sintomas que interferem no consumo alimentar e a hemoglobina (usual/inicial/final) em pacientes com câncer atendidos no ambulatório de oncologia em GuarapuavaPR, vinculado ao Sistema Único de Saúde. Participaram do estudo 147 pacientes, sendo o câncer de mama o mais prevalente (45,6\%). A caquexia esteve presente em diferentes graus no momento do diagnóstico ao final do tratamento. Ao agrupar por tipos de câncer no período inicial ao tratamento o câncer de estômago foi o que apresentou maior percentual de caquexia terminal $(n=5,31,3 \%)$ e o câncer de mama o que apresentou maior percentual de ganho de peso $(n=25 ; 37,3 \%)$, com consequente redução dos indivíduos com caquexia. No período final do tratamento os pacientes com câncer de estômago mantiveram a maior prevalência de caquexia terminal (n=7; 43,8\%) e o câncer de mama o que apresentou maior ganho de peso $(n=39 ; 58,2 \%)$. Os sintomas de impacto nutricional mais frequentes foram xerostomia $(n=84 ; 57,1 \%)$ e constipação intestinal $(n=33 ; 22,4 \%)$. Entre os períodos, pacientes com câncer de estômago apresentaram maior percentual de anemia, respectivamente $(n=10 ; 62,5 \% ; n=11 ; 68,8 \%)$, e somente os pacientes com câncer de mama que apresentaram diferença significante $(p=0,01)$. Evidenciou-se no estudo grande prevalência de caquexia, frequente incidência de anemia e de sintomas de impacto nutricional.
\end{abstract}

Palavras-chave: Câncer. Caquexia. Perda de peso. Avaliação nutricional. Anemia.

\begin{abstract}
Nutritional Impact Symptoms (NIS) and anemia are physiological changes commonly observed in cancer patients at earlier the diagnosis and during the radiotherapy and chemotherapy therapy that may interfere in general condition and cancer cachexia incidence. The goal of this study was evaluate cachexia, NIS and anemia incidence in cancer patients. It was evaluated, weight symptoms that interfere in food consumption and hemoglobin (usual/initial/ending period) in cancer patients attended in oncology clinic at Guarapuava-PR, linked to Brazil's publicly funded health care system (SUS). It was enrolled 147 patients, and breast cancer was more prevalent (45.6\%). Cachexia has been present in varying degrees at the diagnosis to treatment ending. Grouping by cancer type in treatment initial period, stomach cancer has shown the highest terminal cachexia percentage $(n=5,31.3 \%)$ and breast cancer presented highest weight gain percentage $(n=25 ; 37.3 \%)$ with consequent reduction in cachexia subjects. At the treatment ending, stomach cancer patients remained highest terminal cachexia prevalence $(\mathrm{n}=7 ; 43.8 \%)$ and breast cancer presented highest weight gain $(n=39 ; 58.2 \%)$. The most frequent NIS were xerostomia $(n=84,57.1 \%)$ and constipation $(n=33,22.4 \%)$. Between periods, stomach cancer patients have had higher of anemia percentage, respectively $(n=10,62.5 \% ; n=11$; $68.8 \%)$, and only breast cancer patients have had significant difference $(p=0.01)$. It was demonstrated in this study that greater cachexia degree is the most common incidence of anemia and symptoms of nutritional impact. It was evident in this study, high cachexia prevalence, frequent anemia and nutritional impact symptoms incidence.
\end{abstract}

Keywords: Cancer. Cachexia. Weight loss. Nutritional Assessment. Anemia.

DOI: 10.15343/0104-7809.20164003353361

\footnotetext{
*Universidade Estadual do Centro-Oeste, Campus CEDETEG. Guarapuava - PR, Brasil.

** Hospital de Caridade São Vicente de Paulo. Guarapuava - PR, Brasil.

O autores declaram não haver conflitos de interesses.
} 


\section{INTRODUÇÃO}

De acordo com os dados do Instituto Nacional do Câncer (INCA), estima-se para o biênio de 2016-2017 a ocorrência para o Brasil de aproximadamente 600 mil novos casos de câncer ${ }^{1}$, um aumento em aproximadamente biênio de 2014-2015.

Um dos principais sinais da presença de neoplasia maligna no individuo é a perda de peso involuntária, e superior a $5 \%$ do peso corporal habitual de forma rápida. Essa intensa perda de peso é denominada caquexia, a qual é considerada uma síndrome multifatorial em que no estado patológico determina a perda de proteínas e gordura corporal, manifestando-se como característica principal o emagrecimento, assim, afetando o estado funcional, prejudicando o sistema imunológico, causando disfunções metabólicas (anorexia), menor resposta ao tratamento quimioterápica e alta mortalidade ${ }^{2}$.

O metabolismo anormal observado durante a caquexia no câncer leva à mobilização de proteínas musculares e dos estoques de gorduras, afetando a massa corporal. Sendo assim, os fatores presentes na caquexia em pacientes oncológicos que causam a depleção do estado nutricional estão relacionados com o aumento metabolismo energético ${ }^{3}$. Neste pacientes a redução da ingestão de nutrientes e o aumento das necessidades energéticas contribuem para redução das reservas nutricionais, devido ao metabolismo das células tumorais e a também a própria localização do câncer pode influenciar no consumo alimentar e contribuir para a perda de peso ${ }^{4}$.

Os pacientes com câncer frequentemente apresentam sintomas de impacto nutricional (SIN), que são alterações fisiológicas provocadas pelo próprio câncer e/ou seus tratamentos (cirúrgico ou radio/quimioterápico) que limitam ou impedem o paciente se alimentar ${ }^{5}$. Pacientes com caquexia apresenta maior incidência de sintomas de impacto nutricional (SIN) antes diagnóstico e durante o tratamento da radioterapia e quimioterapia dentre os quais, os mais comuns são; náuseas, vômitos, diarreia, saciedade precoce, má-absorção, obstipação intestinal, xerostomia, disfagia, desta forma afetando negativamente o estado nutricional ${ }^{6}$.

Outro achado frequente em pacientes com câncer é a alta incidência de anemia, presente em mais de $40 \%$ dos casos, e em pacientes durante a quimioterapia, a incidência pode elevar-se a $90 \%{ }^{7}$. Desta forma, quando presente, a anemia exerce uma influência negativa na qualidade de vida de pacientes com câncer, sendo identificada como um fator prognóstico desfavorável ${ }^{8}$.

A avaliação nutricional possibilita identificar o risco nutricional do indivíduo com câncer, identificando a perda de peso, os fatores que podem afetar o consumo alimentar e assim podem melhorar o prognóstico do paciente com a intervenção precoce e no momento oportuno ${ }^{9}$. Nesse contexto o objetivo deste estudo em avaliar a perda de peso, o estado nutricional, a prevalência de caquexia, sintomas de impacto nutricional e incidência de anemia em pacientes oncológicos antes e após o tratamento quimioterápico.

\section{MATERIAIS E MÉTODOS}

Estudo longitudinal retrospectivo realizado no período de junho de 2013 a maio de 2014, em pacientes atendidos pelo serviço de nutrição no Ambulatório de Oncologia na cidade de Guarapuava - PR, aprovado no Comitê de Ética em Pesquisa - COMEP/UNICENTRO, sob o protocolo de pesquisa de número 247.385 de 2013.

Os dados iniciais à quimioterapia foram coletados do prontuário de atendimento nutricional dos pacientes: localização primária do câncer, gênero, idade, altura, peso usual (PU - precedente ao diagnóstico informado pelo paciente), peso inicial (PI - antes do tratamento quimioterápico) e 15 sintomas de impacto nutricional (SIN): anorexia, aversão alimentar, alteração de olfato, alteração de paladar, constipação, dor abdominal, diarreia, disfagia, esofagite, flatulência, intolerância a lactose, mucosite, náuseas, vomito e xerostomia. Os dados de peso final (PF - do último ciclo de quimioterapia) e hemoglobina foram coletados do prontuário do tratamento médico/ quimioterápico/ambulatorial no mês de maio de 2014. 
Informações referentes ao estadiamento do tumor foram coletados do prontuário, sendo que a classificação utilizada pelo serviço está de acordo com a classificação proposta pelo sistema TNM (Classificação de Tumores Malignos $6^{\underline{a}}$ ed. INCA 2004).

$\mathrm{O}$ estado nutricional foi determinado pelo cálculo do Índice de Massa Corporal (IMC, $\mathrm{Kg} / \mathrm{m} 2$ ) para os momentos usual, inicial e final, sendo classificados, conforme a Organização Mundial da Saúde ${ }^{10}$. Foi calculada a perda de peso inicial (PPI) e final (PPF) (sendo o peso usual - peso inicial ou peso final), percentual de mudança de peso inicial (\%MPI) e final (\%MPF) (sendo o peso usual/peso inicial ou peso final $x$ $100 \%$ ). De acordo com o percentual de perda de peso os pacientes foram classificados como: ganho, quando tiveram percentual positivo; estável, quando não mudaram o percentual e quando foi observado o percentual negativo e para estadiar caquexia e anemia (hemoglobina) foram estratificados de acordo com o ponto de corte sugeridos por Argilés et al. $(2011)^{11}$.

\begin{tabular}{|lc|}
\hline $\begin{array}{l}\text { Estadiamento da Caquexia } \\
\text { de acordo com o \% } \\
\text { de Perda de Peso Corporal }\end{array}$ & Valores \\
\hline Pré- Caquexia & $<5 \%$ \\
\hline Leve & $\geq 5 \%$ \\
\hline Moderada & $\geq 10 \%$ \\
\hline Grave & $\geq 15 \%$ \\
\hline Terminal & $\geq 20 \%$ \\
\hline Presença de anemia & $\mathrm{Hb}<12 \mathrm{~g} / \mathrm{dL}$ \\
\hline Hemoglobina
\end{tabular}

Quadro 1- Critérios Casco para o estadiamento da caquexia e anemia (Argilés, 2011), Guarapuava, PR, 2013-2014.

Os dados foram analisados por meio de estatística descritiva com médias, desvio-padrão, frequências relativas e absolutas. A distribuição das variáveis foi verificada por meio do Teste de Kolmogorov-Sminorv. Para comparação das variáveis quantitativas relativas ao período inicial e final do tratamento, foi utilizado o Teste-T para amostras pareadas. Para comparação das variáveis categóricas foi utilizado o Teste do
Qui-quadrado. As análises estatísticas foram realizadas com auxílio do Software SPSS versão 22.0, com nível de significância de 5\% $(p<0,05)$.

\section{RESULTADOS}

Participaram do estudo 147 pacientes oncológicos, com média de idade 57,4 411,3 anos sendo $64,6 \%(n=95)$ do gênero feminino. A maior incidência de localização primária do tumor dos pacientes foi o câncer de mama com 45,6\% ( $n=67)$, seguido do câncer de cabeça/ pescoço e pulmão, ambos com $14,3 \%(n=21)$. O estadiamento de acordo com a Classificação TNM foi T1: 6 (4,1\%), T2: 38 (25,9\%), T3: 28 $(19,0 \%)$, T4: $42(28,6 \%)$ e sem classificação 33 $(22,4 \%)$ pacientes.

Verificou-se continuada perda de peso no momento inicial para o momento final ao tratamento, exceto para o câncer de mama, que apresentaram ganho de peso em todo o período avaliado (Tabela 1).

Relacionado ao estado nutricional verificado por meio do IMC, foi identificado alteração siginificante $(p<0,05)$ na classificação do IMC U para o IMC I no câncer de esôfago, pulmão e mama, antes do diagnóstico. No momento do diagnóstico até ao final do tratamento, o parâmetro IMC I para o IMC F apresentou diferença significante $(p<0,05)$ na classificação do estado nutricional dos pacientes com câncer de cabeça/pescoço, esôfago, pulmão e mama. Salienta-se que pacientes diagnosticados com câncer de mama aumentaram a categorização do IMC decorrente do ganho de peso (Tabela 2).

A caquexia no início para o final do tratamento esteve presente em diferentes graus e diferiu entre os diversos sítios primários dos tumores. Quando classificados com caquexia do leve (perda de peso de $\geq 5 \%$ ) à terminal (perda de peso $\geq 20 \%$ ) no período inicial do tratamento o câncer de estômago foi o que apresentou maior percentual de caquexia terminal $(n=5,31,3 \%)$ e o câncer de mama o que apresentou maior percentual de ganho de peso $(n=25 ; 37,3 \%)$, com consequente redução dos indivíduos com caquexia. No período final 
do tratamento os pacientes com câncer de estômago mantiveram a maior prevalência de caquexia terminal $(n=7 ; 43,8 \%)$ e o câncer de mama o que apresentou maior ganho de peso ( $\mathrm{n}=39 ; 58,2 \%$ ) (Tabela 3).

Os sintomas de impacto nutricional mais relatados pelos pacientes durante a primeira avaliação precedente ao tratamento quimioterápico foi a xerostomia $57,1 \%(n=84)$, seguida da constipação intestinal 22,4\% $(n=33)$ e dores abdominais $19,7 \%(n=29)$.

Portadores de câncer de estômago início do tratamento apresentaram valores de hemoglobina $(\mathrm{HB})$ médio de 11,3 g/dL abaixo do ponto de corte proposto neste estudo, e alta prevalência de anemia (62\%).

Observa-se que os valores $\mathrm{HB}$ foram reduzidos em todos os tipos de câncer no decorrer do tratamento, aumentando o número de pacientes anêmicos.

No período inicial e final do tratamento os indivíduos com câncer de estômago apresentaram maior percentual de anemia, respectivamente $(n=10 ; 62,5 \% ; n=11 ; 68,8 \%)$, sendo que somente os pacientes com câncer de mama que apresentaram diferença significante $(p=0,01)$ entre os períodos em relação ao diagnóstico de anemia. (Tabela 5).

Tabela 1 - Alterações de peso no início durante e no final do tratamento de pacientes oncológicos, Guarapuava, PR, 2013-2014.

\begin{tabular}{|c|c|c|c|c|c|c|}
\hline $\begin{array}{l}\text { Localização } \\
\text { do tumor }\end{array}$ & $\begin{array}{l}\text { Cabeça/ } \\
\text { Pescoço }\end{array}$ & Esôfago & Estômago & Colorretal & Mama & Pulmão \\
\hline n (\%) & $21(14,28)$ & $8(5,47)$ & $16(10,88)$ & $14(9,52)$ & $67(45,57)$ & $21(14,28)$ \\
\hline \multicolumn{7}{|l|}{ Peso (kg) } \\
\hline Usual & $68,06 \pm 7,8$ & $71,50 \pm 14,2$ & $70,44 \pm 13,6$ & $74,64 \pm 11,9$ & $69,30 \pm 13,3$ & $63,17 \pm 14,4$ \\
\hline Inicial & $61,20 \pm 12,8^{\mathrm{a}}$ & $60,88 \pm 13,7^{\mathrm{a}}$ & $60,45 \pm 11,5^{a}$ & $67,59 \pm 11,0^{\mathrm{a}}$ & $69,88 \pm 14,2$ & $57,88 \pm 12,7^{a}$ \\
\hline Final & $61,31 \pm 14,6^{b}$ & $60,31 \pm 16,2^{b}$ & $58,06 \pm 10,8^{b}$ & $69,11 \pm 13,0^{b}$ & $70,96 \pm 14,6$ & $56,19 \pm 11,4^{b}$ \\
\hline \multicolumn{7}{|l|}{ IMC (kg/m2) } \\
\hline Usual & $24,7 \pm 2,8$ & $25,6 \pm 5,1$ & $26,04 \pm 4,8$ & $27,40 \pm 3,6$ & $27,20 \pm 5,3$ & $23,48 \pm 3,9$ \\
\hline Inicial & $22,0 \pm 3,5^{c}$ & $21,8 \pm 4,9^{c}$ & $22,34 \pm 3,9^{c}$ & $24,77 \pm 3,0^{c}$ & $27,41 \pm 5,6$ & $21,61 \pm 4,0^{c}$ \\
\hline Final & $21,9 \pm 4,2^{\mathrm{d}}$ & $21,5 \pm 5,7^{d}$ & $21,44 \pm 3,6^{d}$ & $25,28 \pm 3,8^{d}$ & $27,80 \pm 5,6$ & $21,02 \pm 3,8^{\mathrm{d}}$ \\
\hline \multicolumn{7}{|l|}{$\begin{array}{l}\text { Mudança } \\
\text { de Peso (kg) }\end{array}$} \\
\hline Inicial & $-6,9 \pm 10,1$ & $-10,6 \pm 8,6$ & $-9,9 \pm 11,6$ & $-7,0 \pm 6,4$ & $0,58 \pm 7,2$ & $-5,2 \pm 8,1$ \\
\hline Final & $-6,7 \pm 12,0$ & $-11,1 \pm 9,2$ & $-12,3 \pm 11,4$ & $-5,5 \pm 7,1$ & $1,7 \pm 9,2$ & $-7,0 \pm 9,9$ \\
\hline \multicolumn{7}{|l|}{$\begin{array}{l}\% \text { de Mudança } \\
\text { de Peso }\end{array}$} \\
\hline Inicial & $10,1 \pm 14,8$ & $14,5 \pm 10,3$ & $12,9 \pm 14,5$ & $9,2 \pm 8,0$ & $+1,2 \pm 9,9$ & $7,6 \pm 12,4$ \\
\hline Final & $10,0 \pm 17,5$ & $16,0 \pm 12,9$ & $16,5 \pm 12,9$ & $7,5 \pm 9,8$ & $+3,1 \pm 13,2^{\mathrm{e}}$ & $9,7 \pm 13,9$ \\
\hline
\end{tabular}

Nota: Número amostral; \%: percentual; IMC: Índice de Massa Corporal; Kg/m2: quilos por metros quadrados; - Teste t pareado $\mathrm{p}<0,05$ - aPeso $U$ x Peso I, bPeso U x Peso F, cIMC U x IMC I, dIMC U x IMC F e e\% MPI x MPF. 
Tabela 2 - Comparação do diagnóstico do IMC usual, inicial e final, de pacientes oncológicos, Guarapuava, PR, 2013-2014.

\begin{tabular}{|c|c|c|c|c|c|c|}
\hline $\begin{array}{c}\text { Localização } \\
\text { do tumor }\end{array}$ & $\begin{array}{l}\text { Cabeça/ } \\
\text { Pescoço }\end{array}$ & Esôfago & Estômago & Colorretal & Mama & Pulmão \\
\hline \multicolumn{7}{|l|}{$\begin{array}{l}\text { IMC Usual - } \\
\text { n (\%) }\end{array}$} \\
\hline Baixo Peso & $1(4,8)$ & $1(12,5)$ & $1(6,3)$ & - & $1(1,5)$ & $4(19,0)$ \\
\hline Eutrofia & $11(52,4)$ & $3(37,5)$ & $7(43,8)$ & $5(35,7)$ & $26(38,8)$ & $8(38,1)$ \\
\hline Sobrepeso & $9(42,9)$ & $3(37,5)$ & $4(25,0)$ & $5(35,7)$ & $21(31,3)$ & $8(38,1)$ \\
\hline Obesidade & - & $1(12,5)$ & $4(25,0)$ & $4(28,6)$ & $19(28,4)$ & $1(4,8)$ \\
\hline \multicolumn{7}{|l|}{$\begin{array}{l}\text { IMC Inicial - } \\
\text { n (\%) }\end{array}$} \\
\hline Baixo Peso & $6(28,6)$ & $1(12,5)$ & $5(31,3)$ & - & $1(1,5)$ & $4(19,0)$ \\
\hline Eutrofia & $12(57,1)$ & $6(75,0)$ & $5(31,3)$ & $9(64,3)$ & $25(37,3)$ & $12(57,1)$ \\
\hline Sobrepeso & $3(14,3)$ & - & $6(37,5)$ & $4(28,6)$ & $21(31,3)$ & $5(23,8)$ \\
\hline Obesidade & - & $1(12,5)$ & - & $1(7,1)$ & $20(29,9)$ & - \\
\hline \multicolumn{7}{|l|}{$\begin{array}{l}\text { IMC Final - } \\
\text { n (\%) }\end{array}$} \\
\hline Baixo Peso & $6(28,6)$ & $2(25,0)$ & $2(12,5)$ & $1(7,1)$ & - & $5(23,8)$ \\
\hline Eutrofia & $10(47,6)$ & $5(62,5)$ & $11(68,8)$ & $7(50,0)$ & $23(34,3)$ & $11(52,4)$ \\
\hline Sobrepeso & $4(19,0)$ & - & $3(18,8)$ & $4(28,0)$ & $24(35,8)$ & $5(23,8)$ \\
\hline Obesidade & $1(4,8)$ & $1(12,5)$ & - & $2(14,3)$ & $20(29,9)$ & - \\
\hline IMC U x IMC I\# & $p=0,505$ & $p=0,014$ & $p=0,291$ & $p=0,315$ & $p=0,000$ & $p=0,016$ \\
\hline IMC I x IMC F\# & $p=0,000$ & $p=0,023$ & $p=0,033$ & $p=0,131$ & $p=0,000$ & $p=0,000$ \\
\hline
\end{tabular}

Nota: IMC : Índice de massa corporal; n: número amostral; \% : percentual; \#Teste Qui-quadrado de Pearson $\mathrm{p}<0,05$

Tabela 3 - Classificação da caquexia no Início e no Final do tratamento, Guarapuava, PR, 2013-2014.

\begin{tabular}{lcccccc}
\hline & $\begin{array}{c}\text { Cabeça/ } \\
\text { Pescoço }\end{array}$ & Esôfago & Estômago & Colorretal & Mama & Pulmão \\
\hline INICIAL n (\%) & & & & & \\
\hline Caquexia Terminal & $5(23,8)$ & $2(25,0)$ & $5(31,3)$ & $2(14,3)$ & $3(4,5)$ & $3(14,3)$ \\
Caquexia Severa & $1(4,8)$ & $1(12,5)$ & $3(18,8)$ & $1(7,1)$ & $1(1,5)$ & $1(4,8)$ \\
Caquexia Moderada & $5(23,8)$ & $2(25,0)$ & $2(12,5)$ & $4(28,6)$ & $1(1,5)$ & $6(28,6)$ \\
Caquexia Leve & $1(4,8)$ & $1(12,5)$ & $1(6,3)$ & $3(21,4)$ & $2(3,0)$ & $1(4,8)$ \\
Pré-Caquexia & $4(19,0)$ & $2(25,0)$ & $1(6,5)$ & $2(14,3)$ & $10(14,9)$ & $4(19,0)$ \\
Estável & - & - & - & $1(7,1)$ & $25(37,3)$ & $1(4,8)$ \\
Ganho & $5(23,8)$ & - & $5(31,3)$ & $1(7,1)$ & $25(37,3)$ & $5(23,8)$ \\
\hline
\end{tabular}


...continuação - Tabela 3

358

\begin{tabular}{lcccccc}
\hline FINAL n (\%) & \multicolumn{1}{l}{} \\
\hline Caquexia Terminal & $6(28,6)$ & $3(37,5)$ & $7(43,8)$ & $2(14,3)$ & $3(4,5)$ & $5(23,8)$ \\
Caquexia Severa & $3(14,3)$ & $2(25,0)$ & $1(6,3)$ & $2(14,3)$ & $3(4,5)$ & $3(14,3)$ \\
Caquexia Moderada & $3(14,3)$ & $1(12,5)$ & $2(12,5)$ & $1(7,1)$ & $4(6,0)$ & $4(19,0)$ \\
Caquexia Leve & $1(4,8)$ & - & $1(6,3)$ & $1(7,1)$ & $2(3,0)$ & $2(9,5)$ \\
Pré-Caquexia & $2(9,5)$ & $1(12,5)$ & - & $4(28,6)$ & $10(14,9)$ & $2(9,5)$ \\
Estável & - & - & - & $2(14,3)$ & $6(9,0)$ & - \\
Ganho & $6(28,6)$ & $1(12,5)$ & $5(31,3)$ & $2(14,3)$ & $39(58,2)$ & $5(23,8)$ \\
\hline Inicial X Final\# & $\mathrm{p}=0,005$ & $\mathrm{p}=0,286$ & $\mathrm{p}=0,141$ & $\mathrm{p}=0,586$ & $\mathrm{p}=0,000$ & $\mathrm{p}=0,130$ \\
\hline
\end{tabular}

Nota: n: número amostral: \% percentual, \#Teste Qui-quadrado de Pearson

Tabela 4 - Sintomas de impacto nutricional em indivíduos antes da Quimioterapia, Guarapuava, PR, 2013-2014.

\begin{tabular}{lcc}
\hline \multicolumn{1}{c}{ Sintomas de Impacto Nutricional - SIN } & $\mathbf{n}$ & \% \\
\hline Xerostomia & 84 & 57,1 \\
Constipação & 33 & 22,4 \\
Dor abdominal & 29 & 19,7 \\
Esofagite & 22 & 15,0 \\
Náuseas & 20 & 13,6 \\
Alteração do paladar & 18 & 12,2 \\
Mucosite & 16 & 10,9 \\
Alteração de Olfato & 15 & 10,2 \\
Vômitos & 11 & 7,5 \\
Flatulência & 11 & 7,5 \\
Disfagia & 4 & 2,7 \\
Intolerância à lactose & 2 & 1,4 \\
Diarreia & 1 & 0,7 \\
Anorexia & 0 & 0 \\
\hline
\end{tabular}

Notas: $\mathrm{n}=$ número amostral; $\%=$ percentual

Tabela 5 - Hemoglobina e anemia em pacientes oncológicos no Início e ao Final do tratamento, Guarapuava, PR, 2013-2014.

\begin{tabular}{lcccccc}
\hline & $\begin{array}{c}\text { Cabeça/ } \\
\text { Pescoço }\end{array}$ & Esôfago & Estômago & Colorretal & Mama & Pulmão \\
\hline HB Inicial $(\mathrm{g} / \mathrm{dL})$ & $13,1+2,4$ & $13,3+1,3$ & $11,3+1,9$ & $12,5+1,8$ & $13,5+1,4$ & $13,1+1,7$ \\
HB Final $(\mathrm{g} / \mathrm{dL})$ & $12,0+1,4^{*}$ & $11,7+2,3^{*}$ & $11,0+2,0$ & $12,3+2,0$ & $12,3+1,1^{*}$ & $11,7+1,5^{*}$ \\
\hline
\end{tabular}

continua... 


\begin{tabular}{|c|c|c|c|c|c|c|}
\hline \multicolumn{7}{|l|}{$\begin{array}{l}\text { Anemia Inicial - } \\
\text { n (\%) }\end{array}$} \\
\hline Anemia & $6(28,6)$ & $1(12,5)$ & $10(62,5)$ & $7(50,0)$ & $7(10,4)$ & $4(19,0)$ \\
\hline Sem anemia & $15(71,4)$ & $7(87,5)$ & $6(37,5)$ & $7(50,0)$ & $60(89,6)$ & $17(81,0)$ \\
\hline $\begin{array}{l}\text { Anemia Final - } \\
\text { n (\%) }\end{array}$ & - & - & - & $1(7,1)$ & $25(37,3)$ & $1(4,8)$ \\
\hline Anemia & $8(38,1)$ & $4(50,0)$ & $11(68,8)$ & $5(35,7)$ & $27(40,3)$ & $13(61,9)$ \\
\hline Sem anemia & $13(61,9)$ & $4(50,0)$ & $5(31,3)$ & $9(64,3)$ & $40(59,7)$ & $8(38,1)$ \\
\hline $\begin{array}{l}\text { Anemia Inicial X } \\
\text { Final\# }\end{array}$ & $p=0,088$ & $p=0,285$ & $p=0,210$ & $p=0,094$ & $p=0,010$ & $=0,081$ \\
\hline
\end{tabular}

Nota: n: número amostral; \%: percentual; HB: Hemoglobina. Teste t pareado $\mathrm{p}<0,05 * \mathrm{Hbl} \times \mathrm{HbF}$; \#Teste Qui-quadrado de Pearson

\section{DISCUSSÃO}

O câncer e estado nutricional estão diretamente relacionados, de modo que alterações fisiológicas oriundas da enfermidade podem causar perda de peso e desnutrição, que por sua vez, influenciam negativamente na evolução da doença, resposta ao tratamento e tempo de sobrevida ${ }^{12}$.

Nesse estudo, a maior prevalência foi de câncer de mama com aproximadamente 45\% dos casos. Segundo o INCA, o câncer de mama é o tipo de neoplasia que mais acomete as mulheres em todo o mundo, sendo que a idade, consumo de álcool, excesso de peso continuam sendo os principais fatores de risco ${ }^{1}$.

Observou-se que a perda de peso entre os pacientes estudados ocorreu antes do diagnóstico do câncer (inicial), exceto para o câncer de mama que apresentou ganho de peso. Essa condição para o câncer de mama pode ser justificada decorrente da expansão de líquido extracelular, ocasionando retenção hídrica e mascarando o real estado nutricional ${ }^{13}$.

No presente estudo a prevalência de estádio avançado da doença pode ter influenciado na elevada incidência de caquexia. Frequente percentual de pacientes oncológicos em período de doença avançada ou terminal intensificam a perda de peso com consequente caquexia ${ }^{14,19}$. Para os sintomas de impacto nutricionais $(\mathrm{SIN})$ precedentes à quimioterapia foi identificado que a maioria dos pacientes apresentavam xerostomia, constipação e dores abdominais. Omlin ${ }^{6}$ verificaram em um grupo de pacientes com oncológicos com doença avançada, que os cinco mais frequentes sintomas de impacto nutricional foram alteração de gosto e cheiro $(27 \%)$, constipação $(19 \%)$, dor abdominal (14\%), disfagia $(12 \%)$ e dor epigástrica (10\%). Segundo estudo de Kubrak ${ }^{15}$ a ingestão diminuída de alimentos pode surgir por dificuldades de deglutição, obstrução mecânica do trato gastrointestinal, dor, e mais raramente, por distúrbios psicológicos.

São responsáveis pela perda de peso e desnutrição, assim os SIN estão diretamente relacionados, com a presença, a localização e extensão do tumor e com os sistemas como a disfagia, odinofagia, atraso do esvaziamento gástrico, saciedade precoce, obstipação, náuseas, vômitos, insuficiência respiratória e astenia, assim responsável pela com a perda de peso prévia ao diagnóstico e tratamento.

No presente estudo os casos de anemia aumentaram na maioria dos tipos de câncer exceção apenas para o coloretal, que diminui de $50 \%$ para $35 \%$. Um achado importante foi o aumento da prevalência de anemia nas pacientes com câncer de mama de 10,4\% para 
40,3\%. A anemia é um achado frequente em pacientes com câncer, ocorrendo em > 40\% dos casos.

Em pacientes tratados em quimioterapia, a incidência de anemia pode subir a 90\%, sendo fator de prognóstico desfavorável exercendo influência negativa à qualidade de vida. Ludwig e colaboradores $^{16}$, reportaram anemia em $19,5 \%$ no início do tratamento e para o quinto ciclo de quimioterapia foi para $46,7 \%$ dos pacientes, sendo as principais causas para o aparecimento desta a redução do consumo alimentar e/ou a toxicidade provocada pelo tratamento quimioterápico.

\section{CONCLUSÃO}

Evidenciou-se no estudo que quanto maior o grau de caquexia em período inicial e final ao tratamento, mais frequente será a incidência de anemia e os sintomas de impacto nutricional, sendo identificado no perfil de pacientes com câncer estômago. Porém, o fato de ocorrer ganho de peso entre o período inicial e final ao tratamento pode revelar limitada interpretação em relação a condição nutricional do paciente, visto que nos pacientes com câncer de mama foi observado maior incidência de ganho de peso entre os períodos de tratamento, no entanto foram os pacientes que apresentaram diferença significante em relação aos padrões de anemia.

Nesse sentido, sugere-se ampla abordagem das variáveis de acompanhamento nutricional para melhor manejo dos pacientes oncológicos, com consequente auxilio na resposta terapêutica e minimização de déficits nutricionais.

AGRADECIMENTOS: Fundação Araucária-PR / SESA-PR / MS-Decit/ CNPq. Programa Pesquisa para o SUS: Gestão Compartilhada em Saúde (PPSUS) - Chamada 04/201.

\section{REFERÊNCIAS}

1. INCA. Estimativa 2016: incidência de câncer no Brasil. [Internet]. Ministério da Saúde Instituto Nacional de Cancer José Alencar Gomes da Silva. 2016. 124 p. Available from: http://www.inca.gov.br/estimativa/2014/sintese-de-resultados-comentarios.asp

2. Schiessel DL. Definitions and Tools to Assessing Cancer Cachexia : a Review. Rev Bras Cancerol. 2013;59(4):583-91.

3. Vaughan VC, Martin P, Lewandowski PA. Cancer cachexia: Impact, mechanisms and emerging treatments. J Cachexia Sarcopenia Muscle. 2013;4(2):95-109.

4. Nicolini A, Ferrari P, Chiara M, Fini M, Pagani S, Giampietro O, et al. Malnutrition, anorexia and cachexia in cancer patients : A minireview on pathogenesis and treatment. Biomed Pharmacother [Internet]. Elsevier Masson SAS; 2013;67(8):807-17. Available from: http://dx.doi.org/10.1016/j.biopha.2013.08.005

5. Farhangfar A, Makarewicz M, Ghosh S, Jha N, Scrimger R, Gramlich L, et al. Nutrition impact symptoms in a population cohort of head and neck cancer patients : Multivariate regression analysis of symptoms on oral intake, weight loss and survival. Oral Oncol [Internet]. Elsevier Ltd; 2014;50(9):877-83. Available from: http://dx.doi.org/10.1016/j.oraloncology.2014.06.009

6. Omlin A, Blum D, Wierecky J, Haile SR, Ottery FD, Strasser F. Nutrition impact symptoms in advanced cancer patients: Frequency and specific interventions, a case-control study. J Cachexia Sarcopenia Muscle. 2013;4(1):55-61.

7. Dicato M, Plawny L, Diederich M. symposium article Anemia in cancer. Ann Oncol Off J Eur Soc Med Oncol / ESMO. 2010;21(Supplement 7):167-72.

8. Stasi R, Abriani L, Beccaglia P, Terzoli E, Amadori S. Cancer-Related Fatigue: Evolving Concepts in Evaluation and Treatment. Cancer. 2003;98(9):1786-801.

10. WHO. Obesity: Preventing and managing the global epidemic: Report of a WHO consultation. World Health Organ Tech Rep Ser. 2000; 894:i-xii, 1-253.

11. Argilés JM, López-Soriano FJ, Toledo M, Betancourt A, Serpe R, Busquets S. The cachexia score (CASCO): A new tool for staging cachectic cancer patients. J Cachexia Sarcopenia Muscle. 2011;2(2):87-93.

12. Cerezo L. Diagnóstico del estado nutricional y su impacto en el tratamiento del cáncer. Oncol. 2005;28(3):129-34. 
13. Yaw YH, Shariff ZM, Kandiah M, Mun CY, Yusof RM, Othman Z, et al. Weight changes and lifestyle behaviors in women after breast cancer diagnosis: a cross-sectional study. BMC Public Health [Internet]. BioMed Central Ltd; 2011;11(1):309. Available from: http:// www.biomedcentral.com/1471-2458/11/309

14. Silva MPN da. Síndrome da anorexia-caquexia em portadores de câncer. Rev Bras Cancerol. 2006;52(1):59-77.

15. Kubrak C, Olson K, Jha N, Jensen L, McCargar L, Seikaly H, et al. Nutrition impact symptoms: key determinants of reduced dietary intake, weight loss, and reduced functional capacity of patients with head and neck cancer before treatment. Head Neck. 2010;32:290-300.

16. Ludwig H, Van Belle S, Barrett-Lee P, Birgegård G, Bokemeyer C, Gascón P, et al. The European Cancer Anaemia Survey (ECAS): A large, multinational, prospective survey defining the prevalence, incidence, and treatment of anaemia in cancer patients. Eur J Cancer. 2004;40(15):2293-306. 